\title{
IMPACTOS DO DESCARTE DE MEDICAMENTOS: OLHARES SOBRE O MEIO AMBIENTE E A SAÚDE
}

\author{
DISPOSAL OF MEDICINES IMPACTS: VIEWS ON THE \\ ENVIRONMENT AND HEALTH
}

\author{
Yanara de Figueiredo Alves Campos ${ }^{1}$ \\ Clara Monteiro Leitão ${ }^{2}$ \\ Sheyla Macedo Ribeiro de Oliveira ${ }^{3}$ \\ Maria Luísa Gomes Andrade ${ }^{4}$ \\ Ana Kesia Matias ${ }^{5}$ \\ Milena Nunes Alves de Sousa ${ }^{6}$
}

RESUMO: Introdução: É sabido que o descarte inadequado de medicamentos é um fator causador de problemas em se tratando da saúde pública assim como também do meio ambiente salientando-se principalmente os advindos de serviços de saúde e o uso doméstico de medicamentos que muitas vezes em demasia ou incorreto. Objetivo: Averiguar o descarte incorreto de fármacos e os possíveis impactos no meio ambiente e na saúde pública provenientes de tal ocorrência. Método: Revisão integrativa da literatura, em que foram utilizados 10 artigos, dos quais foram extraídos da base de dados nacionais e internacionais e selecionados a partir do recorte temporal de 2016 a 2020. Resultados: Os achados indicaram que o descarte incorreto tem muitas implicações para a saúde (70,0\%). Conclusão: é concludente a urgência de conscientização e aprendizagem da população sobre os perigos produzidos pelo descarte inadequados de preparações farmacêuticas, pois são muitos danos à saúde e ao meio ambiente devendo ser utilizada logística reversa como uma das soluções para essa situação além, de leis mais punitivas e em longo prazo a conscientização da população.

Palavras chave: Saúde Pública; Preparações Farmacêuticas; Resíduos Sólidos; Logística Reversa; Legislação.

\footnotetext{
1 Estudante de Medicina do Centro Universitário de Patos (UNIFIP), Patos, Paraíba. E-mail: yanaracampos1105@gmail.com.

${ }^{2}$ Estudante de Medicina do Centro Universitário de Patos (UNIFIP), Patos, Paraíba.

${ }^{3}$ Estudante de Medicina do Centro Universitário de Patos (UNIFIP), Patos, Paraíba.

${ }^{4}$ Estudante de Medicina do Centro Universitário de Patos (UNIFIP), Patos, Paraíba.

${ }^{5}$ Estudante de Medicina do Centro Universitário de Patos (UNIFIP), Patos, Paraíba.

${ }^{6}$ Docente no Curso de Medicina do Centro Universitário de Patos (UNIFIP), Patos, Paraíba. E-mail: milenanunes@fiponline.edu.br.
} 
ABSTRACT: Introduction: It is known that the inadequate disposal of medicines is a factor that causes problems when it comes to public health as well as the environment, especially those arising from health services and the domestic use of medicines that are often too much or incorrect. Objective: To investigate the incorrect disposal of drugs and the possible impacts on the environment and public health arising from such occurrence. Method: Integrative literature review, in which 10 articles were used, from which they were extracted from the national and international database and selected from the time frame from 2016 to 2020. Results: The findings indicated that the incorrect disposal has many implications for health (80.0\%). Conclusion: the urgency of the population's awareness and learning about the dangers produced by inadequate disposal of pharmaceutical preparations is conclusive, as there are many damages to health and the environment, and reverse logistics should be used as one of the solutions to this situation, in addition to more punitive and long-term public awareness.

Keywords: Public health; Pharmaceutical preparations; Solid waste; Reverse logistic; Legislation. 


\section{INTRODUÇÃO}

A disposição final de resíduos sólidos na superfície do planeta evidencia-se na atualidade como um dos grandes desafios, e entre eles o de medicamentos se tornando assim um problema de saúde pública, assim como a sua complexidade e periculosidade sendo esses gerados pelas atividades humanas domésticas, comerciais, rurais, industriais e médico-hospitalares, esses resíduos, quando descartados inadequadamente, geraram impactos negativos nas características físicas, químicas e biológicas do meio ambiente no mundo todo, com mais relevância na água e no solo que põem em risco a saúde e a sobrevivência dos seres vivos, sobretudo dos seres humanos (IOB; CAMILO; PETRY, 2013).

A utilização de medicamentos é uma questão social e de saúde publica presente na maioria dos ambientes domésticos. Ressaltando que nesse contexto, existe uma preocupação em relação aos problemas oriundos do uso dessas substâncias, tais como: automedicação, intoxicação, desperdício e descarte incorreto (IOB; CAMILO; PETRY, 2013, p. 9).

Embora ainda hoje não se dê a devida importância em se tratar do descarte adequado para esse tipo de lixo, como o descarte de medicamentos, estes podem causar contaminação do solo e da água se dispostos em locais inadequados, tais como lixões e terrenos abandonados. O descarte de medicamentos (sejam eles de validade expirada, contaminados, interditados ou não utilizados por quaisquer outros motivos), tornou-se um problema de saúde pública e ambiental, problema este, que tem sido cada vez mais agravado devido à ausência de atenção dada pela sociedade, o que faz o problema se agravar cada vez mais de forma silenciosa.

De acordo com a utilização do medicamento e o resíduo remanescente, o saldo pode ser classificado, segundo a sua origem, como Resíduos de Medicamento Domiciliar (RMD) ou de Serviços de Saúde (RSS). Segundo Daughton e Ruhoy (2008), a geração de RMD decorre principalmente da falta de adesão plena ao tratamento bem como da inobservância da forma, quantidade e demais orientações 
prescritas, acarretando na não finalização do mesmo causando doenças recorrentes e, por conseguinte, maior quantitativo de desprezo de medicamentos de forma inadequada, sendo esses, medicamentos gerados diretamente pela população, industrializados ou manipulados, que estão vencidos ou em desuso.

A principal legislação com os princípios, objetivos, instrumentos e diretrizes sobre o descarte no Brasil é a Política Nacional de Resíduos Sólidos (PNRS), Lei 12.305 de 2010 (BRASIL, 2010). A logística reversa está entre suas principais estratégias. $\mathrm{O}$ inc. XII do art. $3^{\circ}$ da PNRS define que a logística reversa busca viabilizar o reaproveitamento ou dar a destinação final ambientalmente adequada aos resíduos. As classes de fabricantes, importadores, distribuidores e comerciantes obrigadas a implantar sistemas de logística reversa, independentemente do serviço de limpeza urbana, estão listadas no art. 33 da PNRS (BRASIL, 2010).

O $\S 1^{\circ}$ do art. 33 da PNRS expressa à preocupação da logística reversa com os impactos dos resíduos para a saúde pública e o meio ambiente. Entretanto, é preciso considerar também o fator socioeconômico do país. Mesmo com um sistema público de saúde ofertante de uma cobertura universal, como o SUS e que não requer pagamento direto pelos pacientes, pois é mantido pelos impostos, o mercado farmacêutico privado e os gastos das famílias com medicamentos são consideravelmente abastados no Brasil quando o relacionamos ao consumo dos mesmos.

O país conta com uma alta prevalência de acesso a medicamentos, do qual se destacam (90\%) os grupos populacionais com maior idade, renda, escolaridade $e$ portadores de doenças crônicas. O uso de medicamentos tem maior relação com as populações do sexo feminino, de maior idade, com cor da pele parda e preta, portadores de doenças crônicas, e maior número de consultas médicas realizadas (BOING et al., 2013, p. 5).

Infelizmente essa cultura é pactuada a anos de que bons médicos são aqueles que medicam, a nossa sociedade sofre ainda de distúrbios mentais com muitas pessoas hipocondríacas a qual aumenta ainda mais esse mercado de consumo exagerado de medicamentos e descarte inadequado dos mesmos, pois 
ainda sofremos com a falta de fiscalização e legislação punitivas para o descarte indevido.

A disposição inadequada de resíduos sólidos é uma das mais comumente reconhecidas formas de poluição do meio ambiente. Como descrito anteriormente, a legislação vidente sobre a Política Nacional de Resíduos Sólidos foi implementada no intuito de gerenciar e administrar de forma adequada os resíduos sólidos, não incluindo os rejeitos radioativos.

O gerenciamento adequado dos resíduos sólidos é de extrema importância para o meio ambiente e consequentemente para saúde das pessoas. Enquanto a Organização Mundial da Saúde (OMS) recomenda a proporção de 1:10.000 farmácias/habitantes, há 3,3:10.000 no Brasil (DOMINGUES et al., 2015). Pelo menos 35,0\% da população (CI95\% 29,0; 40,0; 12 = 83.9\%) se automedica, um valor maior que dos países desenvolvidos e próximos da Colômbia $(27,3 \%)$, Hong Kong (32,5\%), Sudão (28,3\%) e Etiópia (39,2\%).

Projeções do Health Global Report indicam que o Brasil seria o quarto mercado mundial de medicamentos em volume de vendas (US\$ 38.000.000,00 48.000.000,00) em 2017, tornando o Brasil um dos protagonistas dessa problemática. "Em 2014, por exemplo, surpreendentemente, as vendas foram US\$ 65.971.000,00, maiores que as projeções da IMS Health Global Report para 2017, o que representa um volume de 3.154.252.382 medicamentos comercializados" (IMS, 2015).

Uma das complicações do inadequado descarte de resíduos sólidos é a contaminação do solo, que não implica somente no descarte inadequado dos resíduos sólidos, pois depende também da falta de planejamento urbano.

Segundo Moreira et al. (2011), o dietilftalato é originário dos efluentes domésticos domiciliares, concluindo que a poluição foi provocadas pelos próprios seres humanos auto destrutivos que não sabem descartar corretamente seu próprio lixo, não tendo uma atenção maior para essa problemática. Dentro desse contexto, o principal objetivo do presente artigo é analisar o descarte incorreto de fármacos e os impactos no meio ambiente e na saúde pública. 


\section{MATERIAL E MÉTODO}

Revisão integrativa da literatura e para elaborá-la necessitou-se seguir as seguintes etapas: definição da questão norteadora com base na hipótese temática a ser abordada; estabelecimento dos critérios de inclusão e exclusão das publicações; identificação dos estudos pré-selecionado e selecionado e seleção das informações a serem extraídas dos trabalhos selecionados, utilizando um instrumento norteador para auxilio na formulação do banco de dados; avaliação dos estudos inclusos para a revisão/análise dos resultados; interpretação e discussão dos resultados; apresentação da síntese do conhecimento obtido (MENDES; SILVEIRA; GALVÃO, 2008).

A questão norteadora para a elaboração da pesquisa foi: Quais as implicações do descarte inadequado de medicamentos na saúde e no meio ambiente? O referido estudo realizou-se durante os meses de março e abril de 2020, por meio da busca de sobre o tema proposto no período de 2020 a 2016, nas bases de dados do US National Library of Medicine National Institutes of Health (PUBMED), Base de dados em Enfermagem (BDENF), é uma base de dados temática que faz parte do Sistema Latino-Americano e do Caribe de Informação em Ciências da Saúde (LILACS), Biblioteca Eletrônica Científica Online (SCIELO) por meio dos descritores "descartes de medicamentos", "preparações farmacêuticas" e "resíduos", os quais tiveram sua existência confirmada através do site dos Descritores em Ciências da Saúde (DeCS) da Biblioteca Virtual em Saúde(BVS).

A partir dos DeCS citados encontrou-se um total de 6689 trabalhos, os quais foram selecionados a partir dos critérios de inclusão: trazer nos resultados os impactos causados a saúde e ao meio ambiente pelo descarte inadequado de medicamentos; idioma inglês, português ou espanhol; texto completo disponível; tipo de documento artigo; recorte temporal de 2016 a 2020. Já os critérios de exclusão consistiram nos estudos que não estavam ligados ao tema específico e também foram excluídos aqueles que apareceram repetidos. É possível visualizar a filtragem na figura 1. 


\section{Figura 1: Fluxograma da metodologia adotada}

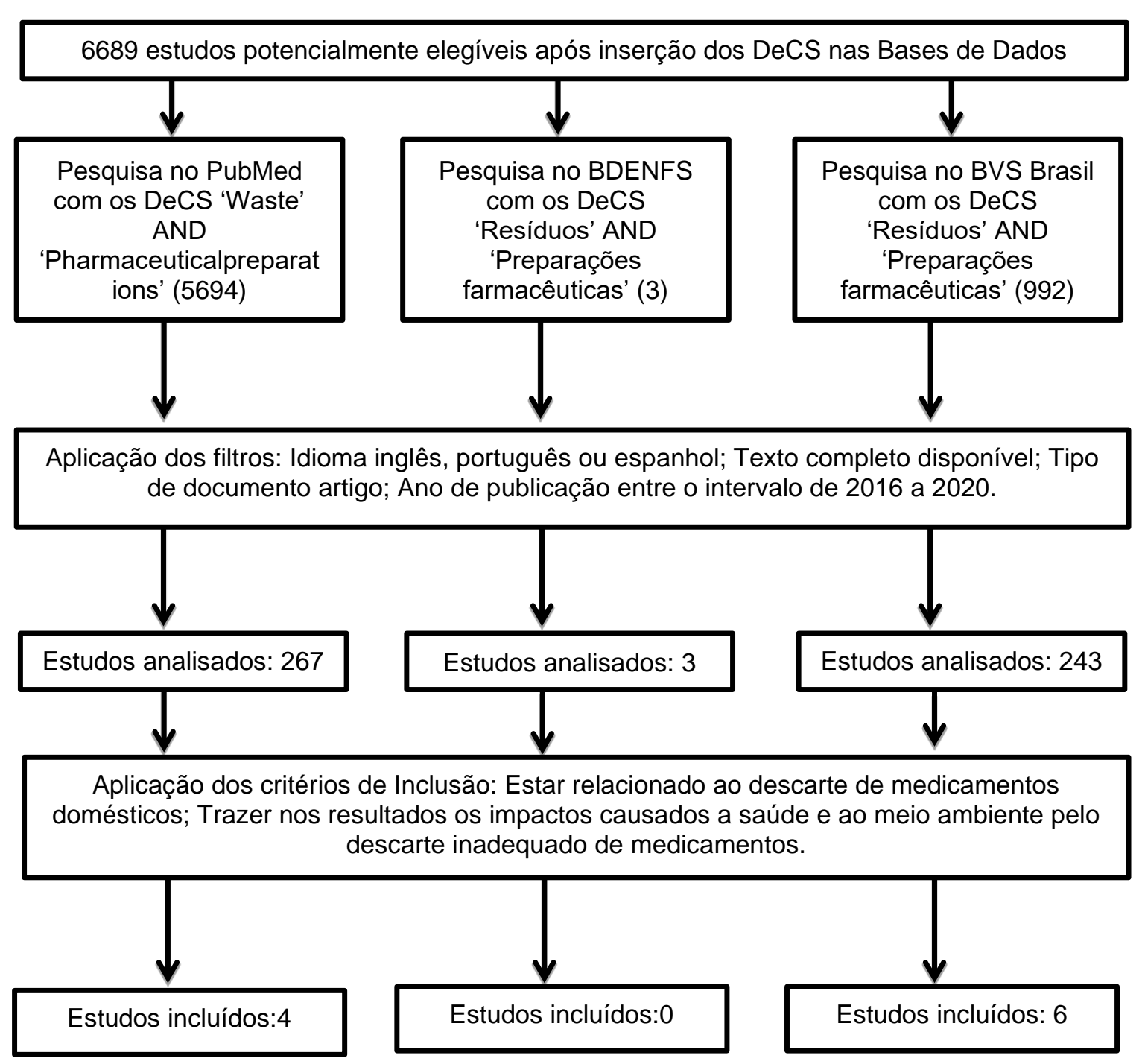

Fonte: Autoria Própria, 2020.

Após a leitura na íntegra das pesquisas, prosseguiu-se com a análise e discussão dos resultados encontrados, as quais ocorreram por meio de descrição em valores absolutos (n) e percentuais (\%) e relação com a literatura pertinente, respectivamente. 


\section{RESULTADOS}

Foram utilizados 10 dos quais foram extraídos da base de dados PUBMED, BDENF e BVS, tratando-se de artigos completos e que foram publicados de 2016 a 2020.

Houve predomínio de estudos encontrados na base de dados do PUBMED, representando $50 \%$ do total, contra $40 \%$ de publicações retiradas da base de dados SCIELO e 10\% publicações do BDENF/LILACS, sendo o idioma principal o inglês $70 \%$, tendo $30 \%$ em português, e o país, o predomínio foi o Brasil em $70 \%$.

\section{Quadro 1: Categorização dos estudos}

\begin{tabular}{|c|c|c|c|c|c|}
\hline Autores/Ano & Título do Artigo & $\begin{array}{l}\text { Base de } \\
\text { Dados }\end{array}$ & Revista & Idioma & País \\
\hline $\begin{array}{l}\text { Bandeira et } \\
\text { al. }(2019)\end{array}$ & $\begin{array}{l}\text { Disposal of } \\
\text { medicines: a socio- } \\
\text { environmental and } \\
\text { health issue }\end{array}$ & $\begin{array}{l}\text { LILACS, } \\
\text { BDENF - } \\
\text { Enfermagem }\end{array}$ & $\begin{array}{l}\text { Revista de } \\
\text { Pesquisa: } \\
\text { Cuidado é } \\
\text { Fundamental } \\
\text { Online }\end{array}$ & Inglês & Brasil \\
\hline $\begin{array}{l}\text { Fernandes et } \\
\text { al. }(2020)\end{array}$ & 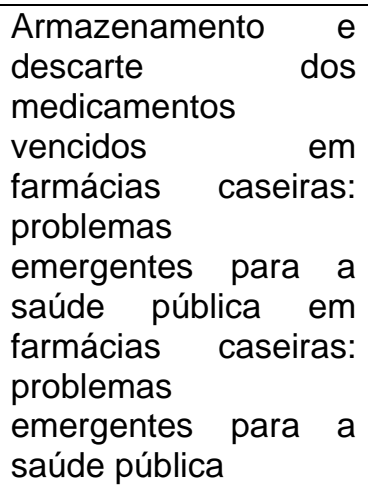 & PUBMED & $\begin{array}{l}\text { Mostra } \\
\text { Científica } \\
\text { Farmácia }\end{array}$ & Português & Brasil \\
\hline $\begin{array}{l}\text { Melo et al. } \\
(2019)\end{array}$ & $\begin{array}{lr}\text { Estudo de } & \text { caso- } \\
\text { gerenciamento } & \text { de } \\
\text { resíduos } & \text { em } \\
\text { farmácias } & \text { de } \\
\text { manipulação de João } \\
\text { Monlevade. }\end{array}$ & $\begin{array}{l}\text { LILACS, } \\
\text { BDENF - } \\
\text { Enfermagem }\end{array}$ & $\begin{array}{l}\text { Observatorio de } \\
\text { la Economía } \\
\text { Latinoamericana }\end{array}$ & Português & Brasil \\
\hline $\begin{array}{l}\text { Athern, } \\
\text { Linnebur, } \\
\text { Fabiasiak } \\
\text { (2016) }\end{array}$ & $\begin{array}{l}\text { Proper disposal of } \\
\text { unused household } \\
\text { medicines: the role of } \\
\text { the pharmacist }\end{array}$ & PUBMED & $\begin{array}{l}\text { Mostra } \\
\text { Científica Da } \\
\text { Farmácia }\end{array}$ & Português & Brasil \\
\hline $\begin{array}{l}\text { Pereira, } \\
\text { Barros } \\
\text { Pereira } \\
\text { (2017) }\end{array}$ & $\begin{array}{l}\text { Pharmacopollution } \\
\text { and Household Waste } \\
\text { Medicine (HWM): how } \\
\text { reverse logistics is } \\
\text { environmentally } \\
\text { important for Brazil }\end{array}$ & PUBMED & $\begin{array}{l}\text { EnvironSciPollut } \\
\text { Res }\end{array}$ & Inglês & Brasil \\
\hline
\end{tabular}




\begin{tabular}{|c|c|c|c|c|c|}
\hline $\begin{array}{l}\text { Quadra et al. } \\
\text { (2017) }\end{array}$ & $\begin{array}{l}\text { Do pharmaceuticals } \\
\text { affect and affect } \\
\text { aquatic ecosystems in } \\
\text { Brazil? A critical } \\
\text { review of current } \\
\text { studies in a } \\
\text { developing country }\end{array}$ & PUBMED & $\begin{array}{l}\text { vironSciPollut } \\
\text { Res }\end{array}$ & Inglês & Brasil \\
\hline $\begin{array}{l}\text { Mohammed } \\
\text { et al. (2016) }\end{array}$ & $\begin{array}{l}\text { Impact of an } \\
\text { educational program } \\
\text { on the knowledge } \\
\text { and practice of the } \\
\text { health team in the } \\
\text { management of } \\
\text { pharmaceutical } \\
\text { waste in Gaza }\end{array}$ & PUBMED & $\begin{array}{l}\text { Palestine } \\
\text { Journal of the } \\
\text { Air \& Waste } \\
\text { Management } \\
\text { Association }\end{array}$ & Inglês & Palestina \\
\hline $\begin{array}{l}\text { Lu et al. } \\
(2016)\end{array}$ & $\begin{array}{l}\text { Occurrence and } \\
\text { efficiency of the } \\
\text { treatment of } \\
\text { pharmaceutical } \\
\text { products in landfill } \\
\text { leached. }\end{array}$ & PUBMED & $\begin{array}{l}\text { Elsevier - West } \\
\text { Management }\end{array}$ & Inglês & Brazil \\
\hline $\begin{array}{l}\text { Al-Shareef et } \\
\text { al. }(2016)\end{array}$ & $\begin{array}{l}\text { Investigating the } \\
\text { disposal of expired } \\
\text { and unused } \\
\text { medicines in Riyadh, } \\
\text { Saudi Arabia: a } \\
\text { cross-sectional } \\
\text { study }\end{array}$ & PUBMED & Int J ClinPharm & Inglês & $\begin{array}{l}\text { Arabia } \\
\text { Saudita }\end{array}$ \\
\hline $\begin{array}{l}\text { Sivasankaran } \\
\text { et al. (2018) }\end{array}$ & $\begin{array}{l}\text { Safe storage and } \\
\text { disposal of unwanted } \\
\text { /unused and expired } \\
\text { medicines: } \\
\text { descriptive cross- } \\
\text { sectional study among } \\
\text { the Rural Indian } \\
\text { population }\end{array}$ & PUBMED & J Young Pharm & Inglês & Índia \\
\hline
\end{tabular}

Fonte: Autoria Própria, 2020.

Sobre a categorização geral dos estudos selecionados observou-se uma maior relevância da população de estudo de pacientes em relação aos trabalhos relacionados aos profissionais de saúde.

\section{Quadro 2: Caracterização geral dos estudos selecionados}

\begin{tabular}{|l|l|}
\hline \multicolumn{1}{|c|}{ Autores/Ano } & \multicolumn{1}{c|}{ População do estudo } \\
\hline Bandeira et al. (2019) & 16 Profissionais da Estratégia de Saúde da Família \\
\hline Fernandes et al. (2020) & $\begin{array}{l}\text { Realizado com 423 usuários de 15 unidades de saúde da } \\
\text { Atenção Primária em um município brasileiro. }\end{array}$ \\
\hline Melo et al. (2019) & Profissionais de farmácias de manipulação. \\
\hline $\begin{array}{l}\text { Athern, } \\
\text { (2016) }\end{array}$ & FinneburFabisiak. \\
\hline
\end{tabular}




\begin{tabular}{|l|l|}
\hline Pereira, Barros e Pereira (2017) & Artigos acadêmicos disponíveis online \\
\hline Quadra et al. (2017) & Artigos acadêmicos \\
\hline Mohammed et al. (2016) & 69Profissionais de Saúde \\
\hline Lu et al. (2016) & Amostras de lixos de aterros. \\
\hline Al-Shareef et al. (2016) & $\begin{array}{l}\text { 50 Pacientes do Hospital Universitário King Khalid } \\
\text { (KKUH), e Rei SaudUniversity (KSU) - Arábia Saudita }\end{array}$ \\
\hline Sivasankaran et al. (2018) & $\begin{array}{l}\text { Uma amostra aleatória de famílias residentes em zonas } \\
\text { rurais indianas }\end{array}$ \\
\hline
\end{tabular}

Fonte: Autoria Própria, 2020.

De acordo com a categorização, houve predomínio da categoria I- Impactos sobre a saúde, com $70,0 \%$ das publicações.

Quadro 3: Categorização dos estudos selecionados.

\begin{tabular}{|c|c|c|c|c|}
\hline Categorias & Subcategorias & Autores/Ano (rever ABNT) & $\mathbf{N}$ & $\%$ \\
\hline \multirow[b]{2}{*}{$\begin{array}{l}\text { Categoria I- } \\
\text { Impactos sobre } \\
\text { a saúde }\end{array}$} & $\begin{array}{c}\text { Contaminação por } \\
\text { exposição aos } \\
\text { medicamentos descartados }\end{array}$ & $\begin{array}{c}\text { Athern, Linnebur; Fabisiak (2016) } \\
\text { Bandeira et al. (2019) } \\
\text { Melo et al. (2019) } \\
\text { Mohammed et al. (2016) } \\
\text { Sivasankaran et al. (2018) }\end{array}$ & 5 & 20 \\
\hline & $\begin{array}{l}\text { Intoxicação/infecção da } \\
\text { população por exposição } \\
\text { aos medicamentos } \\
\text { descartados }\end{array}$ & $\begin{array}{c}\text { Al-Shareef et al. (2016) } \\
\text { Athern; Linnebur; Fabisiak (2016) } \\
\text { Bandeira et al., 2019) } \\
\text { Fernandes et al. (2020) } \\
\text { Lu et al. (2016) } \\
\text { Melo et al. (2019) } \\
\text { Mohammed et al. (2016) } \\
\text { Pereira, Barros e Pereira (2017) } \\
\text { Quadra et. al. (2017) } \\
\text { Sivasankaran et al. (2018) } \\
\end{array}$ & 10 & 50 \\
\hline \multirow{2}{*}{$\begin{array}{l}\text { Categoria II- } \\
\text { Impactos sobre } \\
\text { o meio } \\
\text { ambiente }\end{array}$} & $\begin{array}{c}\text { Intoxicação da água com } \\
\text { excesso de medicação } \\
\text { descartada em afluentes e } \\
\text { oceano; }\end{array}$ & $\begin{array}{c}\text { Bandeira et al. (2019) } \\
\text { Lu et al. (2016) } \\
\text { Mohammed et al. (2016) }\end{array}$ & 3 & 15 \\
\hline & $\begin{array}{l}\text { Contaminação do solo por } \\
\text { resíduos inadequadamente } \\
\text { descartados }\end{array}$ & $\begin{array}{c}\text { Melo et al. (2019) } \\
\text { Quadra et al. (2017) }\end{array}$ & 2 & 5 \\
\hline \multicolumn{3}{|l|}{ Total } & 20 & 100 \\
\hline
\end{tabular}

Fonte: Autoria Própria, 2020. 


\section{DISCUSSÃO}

As atividades que prejudiquem 0 meio ambiente são ampliadas para o descarte inadequado de resíduos, sendo eles inclusive, o desprezo inapropriado de medicamentos, sendo tal habito responsável por dano severo o meio ambiente e a saúde humana (por exemplo, pela contaminação do lençol freático). É importante ressaltar que as ações de reversão dos danos são mais árduas e consideravelmente caras que a prevenção do mesmo, apesar disso, em nível federal, não existe documento Legal que estabeleça critérios rígidos para o descarte de resíduos sólidos.

Isto se relacionado à análise química das amostras ambientais fornece uma medida para a concentração total de substâncias potencialmente nocivas. No entanto, técnicas químicas podem não ser capazes de detectar certos compostos desconhecidos. Avaliação da contaminação e considerável risco tóxico para o meio ambiente das águas residuais não pode ser avaliada por meios químicos sozinhos (LI et al., 2006; PHENRAT, 2007).

Segundo Moreira et al. (2011), uma das regiões com maior incidência é a região metropolitana de Belo Horizonte (MG), por exemplo, se diferencia pelos dados relevantes que situam a presença de farmacopoluentes na água que causam muitos danos à saúde causando problemas na saúde da população e no meio ambiente, tendo como consequências alterações em peixes provocadas por farmacopoluição e uma ampla geração de RMD (FERREIRA; RODRIGUES; SANTOS, 2015).

Em amostras coletadas foram encontrados resquícios de farmacopoluição da bacia do Rio das Velhas (MG) causada pelo hormônio etinilestradiol, na concentração de $4,7 \mathrm{ng} / \mathrm{L}$, quantitativo reconhecido por potencial de geração de danos à saúde de crianças, idosos, se não for devidamente removido. Além daquele hormônio, também foram identificados micropoluentes domésticos estradiol4, o bisfenol A 5, o nonilfenol6 e o dietilftalato7 (PEREIRA; BARROS; PEREIRA, 2017). 
Sendo assim, fica claro que o contato com os agentes contaminantes presentes nos resíduos sólidos pode contaminar os seres vivos por inalação, por contato direto com pele e mucosas, por consumo de água e/ou alimentos contaminados por tais substâncias. Portanto, é muito importante que exista um sistema público de limpeza urbana, que abranja desde a coleta consciente e adequadamente selecionada, tratamento e posterior desprezo dos resíduos sólidos de forma adequada, além de leis mais punitivas e uma maior conscientização da população em longo prazo, pois uma vez que todas as práticas humanas refletem na geração de resíduos, que é um grande desafio para as políticas públicas. Sendo possível intervir para que haja redução considerável dos impactos do descarte desses resíduos para o meio ambiente e para a saúde humana, utilizando um adequado plano de gerenciamento, sendo a logística reversa uma das principais estratégias para ser utilizada.

De acordo com Angonesi e Rennó (2011), deve-se ressaltar que farmacêuticos em cada ambiente têm um papel integral incentivando práticas seguras de eliminação de medicamentos, assim como uma visão multidisciplinar da importante consciência do descarte adequado.

Concernente ao assunto estudado, é mister salientar que outro ponto importante é o armazenamento de medicamentos mediante ao alcance de crianças, sendo reconhecida como principal e mais comum forma de intoxicação em seres humanos no Brasil, conforme o estudo da Universidade Federal de São João DelRei, Divinópolis, MG, Brasil. Foi afirmado que segundo dados do Sistema Nacional de Informações Tóxico Farmacológicas (SINITOX), no período de 2003 a 2012, foram identificados, 22.395 casos de intoxicações, dos quais 17.725 resultaram em hospitalizações e 75 em óbitos, somente em crianças com idade inferior a 5 anos (FERNANDES, 2020).

Diante de análise sobre o descarte de medicamentos pelos profissionais de saúde de Unidades Básica de saúde realizado por Bandeira et al. (2019), observouse que os profissionais avaliados no estudo, identificaram a contaminação do meio ambiente, uso indevido de descartado incorretamente medicamentos e resistência bacteriana a medicamentos como os principais fatores resultantes do descarte incorreto. Nesse sentido, a entrega de um folheto após as entrevistas permitiu que 
os trabalhadores fossem instruídos com as informações corretas sobre como descartar os resíduos e as consequências do descarte incorreto para o meio ambiente e por consequência o ser humano que dele usufrui, sendo uma forma de instiga-los a encontrar mais informações e provocá-los quanto o assunto.

O estudo realizado na Índia, concluiu que a maioria dos entrevistados descarta medicamentos inseguramente. Portanto, os prestadores de cuidados de saúde particularmente farmacêuticos são literalmente melhor equipado para educar o público e criar adequada consciência para armazenamento adequado e descarte seguro de indesejado ou não na medicina preventiva. Além disso, encontrar abordagens para a implementação de leis sobre a destruição de desperdício dos medicamentos é deve ser predominantemente exigida (SIVASANKARAN et al., 2019).

Segundo dessa mesma lógica, em Gaza por meio de um estudo realizado chegou-se a conclusão que se recomenda que as autoridades organizem oficinas sobre PWM regularmente em todos os hospitais e fornecer os recursos necessários para tal gestão sendo a educação a mais eficaz de prevenção já estabelecida (TABASH et al., 2016).

No Brasil, observou-se que com o aumento do uso de drogas, também é extremamente importante educar o público sobre seu uso e descarte correto. Autoridades ambientais brasileiras devem revisar com urgência as leis e fornecer também um controle sobre o desprezo adequado de excedentes farmacológicos. Também é importante aumentar o investimento em tecnologias novas e mais eficazes para o tratamento e remoção desses compostos em corpos d'água. Essa mesma observação se aplicaria a muitos outros países agora enfrentando problemáticas semelhantes (QUADRA et al., 2017).

Diante disso, é importante que, por meio da aplicação da logística reversa, seja realizado o descarte consciente dos resíduos, com o intuito de reciclar ou reaproveitar e fornecer um possível desígnio ambientalmente adequado ao que se considera dentro dos padrões de conservação ambiental, havendo observância de todo potencial de aproveitamento do produto desde o recolhimento do produto até o ponto de consumo final incluindo a sua origem. Para que isso ocorra, deve-se haver um planejamento estratégico das empresas fornecedoras dos produtos em conjunto 
com o governo local, objetivando capacitar e ensinar a população sobre maneiras cabíveis de descarte e meios acessíveis que contenham pontos de descarte de fácil acesso à população, tornando, então, apropriado o manejo correto da logística reversa.

\section{CONCLUSÃO}

É de caráter emergencial a conscientização da população acerca dos perigos produzidos pelo desprezo de resquícios de medicamentos e fármacos decorrentes de compra escusável ou do não cumprimento do esquema terapêutico prescrito pelo médico, podendo trazer doenças até o óbito por intoxicação, como também o câncer pela poluição do meio ambiente (solo, ambiente aquático). Após um programa educacional, estatisticamente melhoria significativa dos hábitos laborais da equipe de saúde e dos usuários no que diz respeito à gerenciamento de resíduos de medicamentos, além de propagarmos mais a estratégia da logística reversa como uma das soluções para essa problemática, incluindo leis mais punitivas.

\section{REFERÊNCIAS BIBLIOGRÁFICAS}

AL-SHAREEF, Fatma et al. Investigating the disposal of expired and unused medication in Riyadh, Saudi Arabia: a cross-sectional study. International Journal Of Clinical Pharmacy, [s.l.], v. 38, n. 4, p. 822-828, 21 mar. 2016.

ANGONESI, D.; RENNÓ, M. U. P. Dispensação farmacêutica: proposta de um modelo para a prática. Ciência e Saúde Coletiva, v. 16, n. 9, p. 3883-3891, 2011.

ATHERN, Kathleen M.; LINNEBUR, Sunny A.; FABISIAK, Greg. Proper Disposal of Unused Household Medications: the role of the pharmacist. The Consultant Pharmacist, v. 31, n. 5, p. 261-266, 1 maio 2016.

BANDEIRA, E. O.; ABREU, D. P. G.; LIMA, J. P.; COSTA, C. F. S.; COSTA, A. R.; MARTINS N. F. F. Descarte de medicamentos: uma questão socioambiental e de saúde. Revista Online de Pesquisa Cuidado é Fundamental, v. 11, n.1, p. 1-10, 2019.

BOING, Alexandra Crispim et al. Acesso a medicamentos no setor público: análise de usuários do Sistema Único de Saúde no Brasil. Cadernos de Saúde Pública, Rio de Janeiro, v. 29, n. 4, p. 691-701, abr. 2013. 
BRASIL. Agência Nacional de Vigilância Sanitária (ANVISA). Resolução RDC no 306, de 07 de dezembro de 2004. Dispõe sobre o Regulamento Técnico para o gerenciamento de resíduos de serviço de saúde. Diário Oficial da República Federativa do Brasil, Brasília, DF, dez. 2004.

BRASIL. Constituição Federal de 1988. Promulgada em 5 de outubro de 1988. Diário Oficial da União, outubro 1988.

BRASIL. Lei $n$. 8.142, de 28 de dezembro de 1990. Dispõe sobre a participação da comunidade na gestão do Sistema Único de Saúde (SUS) e sobre as transferências intergovernamentais de recursos financeiros na área da saúde e dá outras providências. Diário Oficial da União, dezembro 1990b.

BRASIL. Lei $n$. 8080, de 19 de setembro de 1990. Dispõe sobre as condições para a promoção, proteção e recuperação da saúde, a organização e o funcionamento dos serviços correspondentes e dá outras providências. Diário Oficial da União, setembro 1990a.

BRASIL. MINISTÉRIO DA SAÚDE. Agência Nacional de Vigilância Sanitária. Manual de gerenciamento de resíduos de serviços de saúde. Brasília: Ministério da Saúde, 2006.

BRASIL. Ministério da Saúde. Secretaria de Políticas de Saúde. Departamento de Atenção Básica. Política Nacional de Medicamentos (1999). Brasília: Ministério da Saúde, 2002.

BRASIL. Política Nacional de Assistência Farmacêutica (PNAF). Resolução nํㅜ 338, de 06 de maio de 2004. Aprova a Política Nacional de Assistência Farmacêutica. Diário Oficial da União, maio 2004.

BRASIL. Política Nacional de Resíduos Sólidos Urbanos (PNRS), lei 12.305/2010. Brasília, 2010.

BRASIL. Portaria $n . .344$, de 12 de maio de 1998. Aprova o Regulamento Técnico sobre substâncias e medicamentos sujeitos a controle especial. Diário Oficial da União, maio 1998.

BRASIL. Resolução CONAMA no 358, de 29 de abril de 2005. Dispõe sobre o tratamento e a disposição final dos resíduos dos serviços de saúde e dá outras providências. Diário Oficial da União, maio 2005.

DOMINGUES, P. H. F.; GALVÃO, T. F.; ANDRADE, K. R. C.; SÁ, P. T. T.; SILVA, M. T.; PEREIRA, M. G. Prevalência da automedicação na população adulta do Brasil: revisão sistemática. Revista de Saúde Pública, v. 49, n. 36, p. 1-8, 2015.

DOUGHTON, C. G.; RUHOY, I. S. Lower-dose prescribing: Minimizing "side effects" of pharmaceuticals on society and the environment. Science of the Total Environment, v. 443, n. 1, p. 324-337, 2013.

FERNANDES, M. R.; FIGUEIREDO, R. C.; SILVA, L. G. R.; ROCHA, R. S.; BALDONI, A. O. Armazenamento e descarte dos medicamentos vencidos em farmácias caseiras: problemas emergentes para a saúde pública. Einstein (São Paulo), v. 18, n. 1, p. 1-6, 2020.

FERNANDES, Mayra Rodrigues et al. Storage and disposal of expired medicines in home pharmacies: emerging public health problems. Einstein (são Paulo), [s.l.], v. 18, p. 1-6, fev. 2020.

FERREIRA, C. L.; RODRIGUES S. C.; SANTOS M. A. S. Análise do conhecimento da população sobre o descarte de medicamentos em Belo Horizonte - Minas Gerais. Interfaces Científicas - Saúde e Ambiente, v. 3, n.2, p. 9-18, 2015.

IMS. HEALTH DO BRASIL. Report press request. São Paulo: IMS Health do Brasil. 2015. 
IOB, Gelson Antonio; CAMILLO, Elineide Gomes dos Santos; PETRY, Raquel Denise. Análise da forma de descarte de medicamentos por usuários de uma Unidade de Saúde no município de Porto Alegre/RS. Infarma - Ciências Farmacêuticas, v. 25, n. 3, p. 118-125, 30 set. 2013.

LI, L.; FAN, M.; BROWN, R. C.; VAN LEEUWEN, J. H.; WANG, J.; WANG, W.; SONG, Y.; ZHANG, P. Synthesis, properties, and environmental applications of nanoscale ironbased materials: a review. Critical Reviews in Solid State and Materials Sciences, v. 36, n. 5, p. 405-431, 2006.

LU, Mu-chen et al. Occurrence and treatment efficiency of pharmaceuticals in landfill leachates. Waste Management, v. 55, p. 257-264, set. 2016.

MENDES, Karina dalSasso; SILVEIRA, Renata Cristina de Campos Pereira; GALVÃO, Cristina Maria. Revisão integrativa: método de pesquisa para a incorporação de evidências na saúde e na enfermagem. Texto \& Contexto - Enfermagem, v. 17, n. 4, p. 758-764, dez. 2008.

MOHAMMED, I. T. et al. Impact of an educational program on knowledge and practice of health care staff toward pharmaceutical waste management in Gaza, Palestine. Journal Of The Air \& Waste Management Association, v. 66, n. 4, p. 429-438, fev. 2016.

MELO, N. O.; BARROS, A. J.; FERREIRA, T. E.; LIMA, R. P. Estudo de caso-gerenciamento de resíduos em farmácias de manipulação de João Monlevade. Observatório Economía Latinoamericana, v. 1, n. 1, p. 10-15, 2019.

MOREIRA, M.; AQUINO, S.; COUTRIM, M.; SILVA, J.; AFONSO, R. Determination of endocrine-disrupting compounds in waters from Rio das Velhas, Brazil, by liquid chromatography/high resolution mass spectrometry (ESI-LC-IT-TOF/MS). Environmental Technology, v. 32, p. 1409-1417, n. 12, 2011.

MUCELIN, Carlos Alberto; BELLINI, Marta. LIXO E IMPACTOS AMBIENTAIS PERCEPTÍVEIS NO ECOSSISTEMA URBANO. Sociedade \& Natureza, Uberlândia, v. 20, n. 1, p. 111-124, jun. 2008.

PEREIRA, André Luiz. Percepção dos usuários do programa Farmácia de Minas sobre o descarte de resíduos de medicamentos domésticos: uma pesquisa-ação em Betim (MG). 2018.[internet] Disponível em: https://repositorio.ufmg.br/handle/1843/RAOA-BBAKXF. Acesso em:30 Mar. 2020.

PEREIRA, André Luiz; BARROS, Raphael Tobias de Vasconcelos; PEREIRA, Sandra Rosa. Pharmacopollution and Household Waste Medicine (HWM): how reverse logistics is environmentally important to Brazil. Environmental Science And Pollution Research, v. 24, n. 31, p. 24061-24075, 19 set. 2017.

PHENRAT, T.; SALEH, N.; SIRK, K.; TILTON, R. D.; LOWRY, G. V. Aggregation and sedimentation of aqueous nanoscale zerovalent iron dispersions. Environmental Science Technology, v. 41, n. 1, p. 284-290, 2007.

QUADRA, G. R.; H. O.; COSTA, R. S.; FERNANDEZ, M. A. S. Do pharmaceuticals reach and affect the aquatic ecosystems in Brazil? A critical review of current studies in a developing country. Environmental Science and Pollution Research, v. 24, n. 1, p. 1200-1218, 2017.

SIVASANKARAN, P.; MOHAMMED, E. B.; GANESAN, N. DURAI, R. Storage and Safe Disposal of Unwanted/Unused and Expired Medicines: A Descriptive Cross-Sectional Survey among Indian Rural Population. Journal of Young Pharmacists, v. 11, n. 1, p. 97-100, 2019.

SOUSA, Milena Nunes Alves de et al. Estratégias de educação em saúde ambiental: enfoque sobre as experiências brasileiras. In: SOUSA, Milena Nunes Alves de; ALMEIDA, Elzenir 
Pereira de Oliveira (Orgs). Interface Saúde e Meio Ambiente. Curitiba: Appris Editora, 2020.

TABASH, R. A.; HUSSEIN, A. H.; MAHMOUD, M. D. ELBORGY, B. A. Impact of an educational program on knowledge and practice of health care staff toward pharmaceutical waste management in Gaza, Palestine Mohammed I. Journal of the air \& waste management association, v. 66, n. 4, p. 429-438, 2016. 